early 2013, the system vastly overestimated flu cases for two weeks ${ }^{7}$. By relying wholly on associations rather than also incorporating epidemiological risk factors, the algorithm had few checks and balances against over- or underestimation, and offered few insights into the factors driving short-term patterns in flu incidence.

In summary, although big-data analyses cannot replace careful epidemiological studies, they can give broad insight into the potential benefits of public-health policies. In this case, Heft-Neal and colleagues' work highlights the benefits of aspiring to reduce air pollution to the lowest levels observed in their data set, and provides assessments of the effects of more-modest changes in pollution levels. This type of analysis certainly has a place in the modern public-health toolbox. As noted by
Kofi Annan"2:"Without good data, we're flying blind. If you can't see it, you can't solve it." -

Lance A. Waller is in the Department of Biostatistics and Bioinformatics, Rollins School of Public Health, Emory University, Atlanta, Georgia 30322, USA.

e-mail:lwaller@emory.edu

1. Dowell, S. F., Blazes, D. \& Desmond-Hellmann, S. Nature 540, 189-191 (2016).

2. Annan, K. Nature 555, 7 (2018)

3. Heft-Neal, S., Burney, J., Bendavid, E. \& Burke, M. Nature 559, 254-258 (2018).

4. GBD 2016 Mortality Collaborators. Lancet 390, 1084-1150 (2017).

5. Cohen, A. J. et al. Lancet 389, 1907-1918 (2017).

6. Burnett, R. T. et al. Environ. Health Perspect. 122 , 397-403 (2014)

7. Lazer, D., Kennedy, R., King, G. \& Vespignani, A Science 343, 1203-1205 (2014).

This article was published online on 27 June 2018.

\title{
The heat is on for Majorana fermions
}

\section{Exotic particles called Majorana fermions have potential applications in quantum computing, but their existence has yet to be definitively confirmed. Two groups have now glimpsed these particles. SEE ARTICLE P.205 \& LETTER P.227}

\section{KIRILL SHTENGEL}

$\mathrm{T}$ he building-blocks of matter - protons, neutrons and electrons - are examples of particles called fermions. Eight decades ago, the Italian physicist Ettore Majorana predicted the existence of fermions that are their own antiparticles ${ }^{1}$. These particles, now known as Majorana fermions, would be of great fundamental interest, and could revolutionize quantum computing. Evidence for Majorana fermions among elementary particles remains elusive; however, in the past few years, there has been striking progress in this hunt in the realm of condensed-matter physics ${ }^{2}$. On pages 205 and 227, respectively, Banerjee et al. ${ }^{3}$ and Kasahara et al. ${ }^{4}$ report signatures of Majorana fermions in heattransport experiments in two very different condensed-matter settings.

Condensed-matter systems contain excitations that behave like ordinary particles, but that need not resemble the actual elementary particles that the systems are made of. For example, the phenomenon of superconductivity (more specifically, topological superconductivity) provides a setting in which an electron can effectively 'forget' its electric charge. As a result, the electron becomes indistinguishable from its antiparticle, which in this context is an electron vacancy called a hole. Whether topological superconductivity is an intrinsic feature of solid-state materials remains an open question. However, the key aspects of the phenomenon can be mimicked in certain condensed-matter systems, providing the right conditions for the emergence of Majorana fermions. The two systems investigated in the current papers seem to be of just this kind.

Banerjee and colleagues looked for evidence of Majorana fermions on the edge of a condensed-matter system that exhibits the quantum Hall effect - whereby, at low temperature and in the presence of a strong magnetic field, the material's transverse electrical conductance becomes quantized (it can have only specific values). The authors focused on a particular state for which this conductance is $5 / 2$ times the fundamental unit. The exact nature of this state has been a subject of debate, but all of the strong contenders can be thought of as superconducting states of composite fermions ${ }^{5}$.

By contrast, Kasahara and colleagues investigated a form of ruthenium chloride known as $\alpha-\mathrm{RuCl}_{3}$. This material is thought to be in a phase known as the Kitaev spin liquid - a peculiar state of matter that lacks long-range magnetic order all the way down to zero kelvin ${ }^{6,7}$. Although $\alpha-\mathrm{RuCl}_{3}$ is an electrical insulator, the description of the magnetic properties of a Kitaev spin liquid is mathematically equivalent to that of a topological

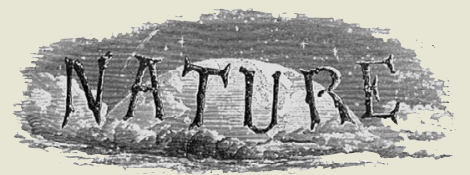

50 Years Ago

Motorists in south and central England who cleaned their cars during the last weekend of June regretted their diligence when they rose on Monday, July 1, to find deposits of orange coloured dust over every exposed surface. The explanation was an early morning shower of rain, laden with dust swept up probably from somewhere in North Africa ... this was an unusual event, even for a country which prides itself on the peculiarities of its climate ... it turned out that the last time a dust fall like this happened was in 1903 ... On the same day as the widespread dust fall, Minehead ... Dulverton ... and Burnley ... were bombarded by hailstones the size of golf balls .. The Meteorological Office is keeping an open mind on whether this is a coincidence or whether there was a causal relationship between the dust and the hail.

From Nature 13 July 1968

100 Years Ago

During the last twenty years there has been an extraordinary increase in the ... output of books and papers on scientific subjects. In the olden time many a quiet student would be content to spend his life upon one piece of work ... in the hope that it might remain a permanent addition to human knowledge ... [A]nyone wishing to learn the present state of our knowledge ... might well despair of ever discovering all that has recently been written ... A complete catalogue of all scientific publications throughout the world would be, unfortunately, very bulky ... An alternative method is to draw up a list of journals ... and to confine the catalogue to papers published in these journals. When this plan is adopted it is hoped that authors ... will gradually acquire the habit of sending any original paper they wish to publish to one of these periodicals. From Nature 11 July 1918 